
\title{
28 Research Suare \\ CT Grading in Upper Urinary Tract Calculi with Kidney Infection
}

\section{Jianping Zhang}

Affiliated Haixia Hospital of Huaqiao University(910th Hospital of PLA)

Rongkai Lin ( $\sim 13905957180 @ 139 . c o m$ )

Affiliated Haixia Hospital of Huaqiao University

Chaoxiong Deng

Affiliated Haixia Hospital of Huaqiao University(910th Hospital of PLA)

Xiaoxia Wu

Prenatal Diagnosis Center, Children's Hospital of Quanzhou

Chaoyu Yang

Affiliated Haixia Hospital of Huaqiao University(910th Hospital of PLA)

\section{Research article}

Keywords: CT, CT values, Upper urinary tract calculi, SIRS

Posted Date: May 8th, 2020

DOI: https://doi.org/10.21203/rs.3.rs-26526/v1

License: (c) (1) This work is licensed under a Creative Commons Attribution 4.0 International License.

Read Full License 


\section{Abstract}

Background This study is to establish a CT imaging grading system and to explore its value in evaluating upper urinary tract calculi with kidney infection.

Methods CT images of 126 patients with kidney infection caused by upper urinary tract calculi were retrospectively analyzed. CT grading system was developed based on CT images. General information, clinical symptoms and examination data of patients in different CT grades were analyzed. With the occurrence of systemic inflammatory response syndrome (SIRS) as the end point, univariate and multivariate analysis was conducted to analyze the risk factors of SIRS.

Results CT images were classified into 4 grades. For patients with different CT grades, the factors of fever, diabetes, blood white blood cell counts, urine leucocytes, CT1 (ratios of renal cortex CT values on the affected side and those on the healthy side), CT2 (ratios of renal pelvis CT values on the affected side and those on the healthy side), maximum body temperature, disease time, proportion of blood neutrophils, C-reactive protein, procalcitonin, and the size of stones had statistical significance $(P<0.05)$. Only CT grading was statistically significant by multivariate analysis. The higher the CT grading, the greater the risk of SIRS, with 4.472 times higher of SIRS risk for each increasement of the CT grade.

Conclusions CT images may reflect the involvement extent and scope of upper urinary tract calculi with kidney infection. The higher the CT grade is, the more severe the clinical symptoms are and the greater the risk of SIRS is.

\section{Introduction}

Upper urinary tract calculi are common in the urinary system. Obstruction caused by upper urinary tract calculi may lead to kidney infection, which may develop into renal abscess if not detected in time or poorly understood, and will lead to septicopyemia if severe [1]. There may be no obvious symptoms even for patients with severe hydronephrosis, which always results in delayed diagnosis and treatment [2]. Currently, the existing imaging examinations mainly focus on the obstruction location of upper urinary tract calculi and the size of the calculi $[3,4]$. However, kidney infection caused by obstruction has not received enough attention. Clinical evaluation of the severity of kidney infection is often based on the symptoms, signs, blood routine examination, inflammatory indicators and other examinations $[5,6]$. The direct and objective imaging diagnostic criteria are lacking.

In recent years, the application of multi-slice spiral CT in kidney diseases has been increasing. CT imaging has not only improved the image resolution, showing more three-dimensional images of kidney anatomy and morphological changes, but also has a marked effect on the detection rate and qualitative diagnosis of kidney diseases $[7,8]$. The main advantages of CT are significant improvement of soft tissue contrast, which may directly and accurately show stones and clearly show tiny stones $[9,10]$. Ureteral obstruction causes urine to accumulate in the kidney or above the ureteral obstruction section, thus causing kidney infections. CT scans can clearly show the extent and characteristics of local 
inflammatory lesions at the ureteral obstruction [11]. However, the clinical diagnosis of obstructive kidney infection is usually based on the patient's symptoms and laboratory tests, but not on CT imaging, which makes it impossible to effectively evaluate the degree and extent of kidney infection.

Systemic inflammatory response syndrome (SIRS) is a systemic inflammatory response caused by infectious or non-infectious factors. It is an immune disorder caused by the excessive release of various inflammatory mediators in the body. If SIRS is not controlled in time, it can progress to sepsis. According to statistics, $30 \%-50 \%$ of SIRS have further developed into septic shock and multiple organ dysfunction syndrome. Therefore, the early diagnosis and treatment of SIRS is very important for the prevention and treatment of sepsis. This is also one of the important issues that urgently need to be solved in clinical urology.

Herein, we aim to investigate the role of a CT imaging grading system in evaluating upper urinary tract calculi with kidney infection. The clinical data of 126 patients with upper urinary calculi caused kidney infection were summarized. A CT imaging grading system was established. The relationship between the CT imaging grading system and clinical symptoms (including SIRS) was analyzed.

\section{Materials And Methods}

\section{Patients}

From April 2013 to October 2019, 126 patients with kidney infection caused by upper urinary tract calculi were enrolled in the study. Inclusion criteria: 1) Patients with upper urinary tract calculi confirmed by CT imaging and clinical diagnosis. 2) Patients did not receive any treatment before enrollment. Exclusion criteria: 1) Patients with end-stage kidney disease at admission. 2) Patients with solitary kidney. 3) Patients with double ureteral calculi. 4) Patients with other systemic infectious diseases. Among the enrolled patients, 50 were males and 76 females. They were aged 27-82, with an average age of 54.2 years. All patients underwent plain CT scan. The lesions were located on the left side in 36 cases and on the right side in 90 cases. The clinical study conformed to the relevant requirements of World Medical Association Declaration of Helsinki. Written informed consents were obtained from every patient and the study was approved by the ethics review board of the 180th Hospital of PLA.

\section{CT examination}

CT Scanning Apparatus Lightspeed VCT XT 64-slice spiral CT scanner and CT quality analysis specialized water phantom were all from GE Healthcare (USA). Before CT examination, the patients were requested to drink water for filling of the bladders. A 64-slice spiral CT scanner was used for plain scanning, in a scan range from diaphragm to pubic symphysis, and, with a thickness of $5 \mathrm{~mm}$ and a reconstruction interval of $1.25 \mathrm{~mm}$. The scanning data was exported to the workstation for the threedimensional reconstruction. The maximum intensity projection, curved planar reformation, volume reformation and multi-planar reconstruction were adopted to display the three-dimensional images and lesions of the urinary tracts. Meanwhile, the relation between lesions and surrounding anatomical 
structures was observed. The images were analyzed by $2 \sim 3$ senior radiologists. According to the scope and extent of inflammation exudation involving the kidneys, CT evaluation criteria were developed and CT images were classified into different grades.

\section{CT grading}

According to the scope and extent of inflammation exudation involving the kidney suggested by CT, we divided the lesions into four grades (Figure 1). In Grade 1 (Figure 1A), the CT signs included thickened ureter wall and vague edema, thickened wall of pelvis and renal calices, mild dilation of pelvis and renal calices, vague fat space of sinus renalis, but not involving renal parenchyma. In Grade 2 (Figure 1B), the CT signs included rough inflammation exudation of kidney capsules, increased density of perirenal fat space, and thickened renal fascias. In Grade 3 (Figure 1C), the CT signs included locally thickened renal parenchyma with decreased density, local wedge-shaped swelling and low-density focuses in renal parenchyma, with the scope less than or equal to $50 \%$. In Grade 4 (Figure 1D), the CT signs included the area of involved renal parenchyma greater than $50 \%$, or with emphysema, or renal and perinephric abscesses.

\section{Indicator measurement and calculation of CT values}

CT images of the lateral axis plane of renal hilum were taken, and the region of interest (ROI) was manually selected to measure the double renal parenchyma value $(\mathrm{Hu})$. ROI were all measured for 3 times and averaged, greater than $100 \mathrm{~mm}^{2}$ for each one. Meanwhile, CT values of bilateral renal pelvis hydrops were measured. The ratios of renal cortex CT values on the affected side and those on the healthy side (CT1), and the ratios of renal pelvis CT values on the affected side and those on the healthy side (CT2) were calculated, and the CT1 and CT2 of different CT grades were compared.

\section{Evaluation Indicators: general data, clinical symptoms and examination data}

General data of patients, including age, gender, and stone location were collected. Clinical symptoms and examination data included fever (body temperature $>38^{\circ} \mathrm{C}$ ), maximum body temperature, renal colic, diabetes, disease time, stone size, CT1, CT2, white blood cells, blood neutrophil percent, C-reactive protein (CRP), procalcitonin (PCT), midstream urine bacterial culture, blood culture and urine leukocytes. All the general data, clinical symptoms and examination data of patients with different CT grades were compared.

\section{Analysis of SIRS risk factors}

The occurrence of systemic inflammatory response syndrome (SIRS) was considered as the end point of observation. The diagnosis of SIRS required at least two criteria as follows: 1 ) body temperature higher than $38^{\circ} \mathrm{C}$ or lower than $36^{\circ} \mathrm{C}$. 2) heart rate over 90 times/min. 3) respiratory rate higher than 20 times /min or arterial blood partial pressure of $\mathrm{CO}_{2}$ lower than $32 \mathrm{mmHg}$ or mechanical ventilation. 4) white blood cell counts higher than $12 \times 10^{9} / \mathrm{L}$ or lower than $4 \times 10^{9} / \mathrm{L}$, or immature white blood cells higher than 
$10 \%$. Univariate and multivariate analysis was conducted to analyze the risk factors of SIRS in patients with upper urinary tract calculi.

\section{Statistical analysis}

SPSS Statistics 23.0 (IBM, American) was used for data analysis. Quantitative data conforming to normal distribution were shown as mean \pm standard deviation (SD). The t-test was used for difference comparison between two groups of independent sample data, and one-way analysis of variance was conducted for data of more than two groups. The quantitative data that did not conform to normal distribution were described by medians (inter-quartile range) and compared by non-parametric test. Chisquare test was adopted for analyzing enumeration data. The correlation analysis between quantitative data and qualitative data was performed by Eta coefficient. Multivariate analysis of dichotomous data was performed by logistic regression analysis. A $p$ value $<0.05$ was considered as statistically significant.

\section{Results}

\section{CT images}

Ureter signs: Ureters above the obstruction plane of the affected side were dilated with hydrops. Ureter wall was slightly thickened. Ureter wall in stone plane were dropsical and vague, and adjacent fascias were thickened.

Signs of renal pelvis: There was thickening of the renal pelvis and calvarial wall. The renal pelvis and calices were dilated. The density of dilated renal pelvis was higher than or equal to that of the healthy side.

Signs of renal parenchyma: Local wedge-shaped swelling diffused from the collecting system to the renal capsules, or in diffuse swelling. The boundaries of the renal cortex and medulla of the swollen part were vague. The swollen part showed slightly high density, and the density of the diseased part was decreased.

Signs of renal capsules and perirenal images: There were rough renal capsules, and thickened perirenal anadesmas and peri-renal space fat, with patchy and banded high density.

\section{Comparison of general data of patients with different CT grades}

The clinical data of patients were shown in Table 1. Based on the CT grading system, there were 48 cases of grade 1 (accounting for $38.1 \%$ ), 30 cases of grade 2 (23.8\%), 24 cases of grade $3(19.0 \%)$ and 24 cases of grade 4 (19.0\%). There were no significant differences in age, sex, side and stone position of patients with different CT grades $(P>0.05)$.

Comparison of clinical symptoms and examination results of patients with different CT grades 
Patients with different CT grades had no statistically significant differences in renal colic, urine bacteria culture positive rate or blood culture positive rate $(P>0.05$, Table 2$)$, but they had statistically significant differences in fever, diabetes, blood WBC counts, urine leucocytes, CT1, CT2, maximum body temperature, disease time, proportion of blood neutrophils, CRP, PCT and the size of stones $(P<0.05$, Table 2). Thus, there was a difference of conditions among patients with different CT grades.

\section{Correlation analysis of clinical symptoms and examination data indicators with CT grades}

Eta coefficient was used to analyze the correlation of clinical symptoms and examination data indicators (including CT1, CT2, CRP and PCT) with CT grades. Using CT grades as the dependent variable, the Eta coefficients of CT grades with CT1, CT2, CRP and PCT were $0.968,0.782,0.965$ and 0.887 , respectively (Table 3), indicating high correlation. When CT1, CT2, CRP and PCT were used as dependent variables, the Eta coefficients of CT grades with CT2 and PCT were less than 0.5, while Eta coefficients of those with CT1 and CRP were both higher than 0.7 , also indicating high correlation.

\section{Analysis of SIRS risk factors}

SIRS occurred in 36 of the enrolled patients. Univariate analysis was used to analyze the risk factors of SIRS. As shown Table 4, the CT1, CT2, CRP, PCT, proportion of neutrophils and CT grades between SIRS group and non-SIRS group was statistically significant $(P<0.05)$. CT1 in the non-SIRS group was significantly higher than that in the SIRS group, and CT2, CT grades, CRP, PCT and proportion of neutrophils in the SIRS group were significantly higher than those in the non-SIRS group. There was no statistical significance in stone size, disease time, blood culture, urine culture, diabetes, and renal colic between the SIRS group and the non-SIRS group $(P>0.05$, Table 4$)$.

In the study, dichotomous logistic regression was used for multivariate analysis. Indicators with significant $P$ values in univariate analysis were included in logistic regression. To avoid missing significant influencing factors, $P$ values were set to be less than 0.1 . Therefore, $C T 1, C T 2, C R P, P C T$, urine culture, neutrophil granulocyte percent and CT grades were used as independent variables in logistic regression analysis. The tolerances were all much greater than 0.1 , and the variance inflation factors were all less than 10 . Thus, there was no multicollinearity.

Finally, the logistic model was statistically significant $(\chi 2=41.380, P<0.001)$. At the same time, the results showed that the model correctly distinguished $90.5 \%$ of the research subjects, with a sensitivity of $82.4 \%$ and a specificity of $93.5 \%$

Among the 7 independent variables included in the model, only CT grade was statistically significant, which was an independent factor influencing SIRS. According to the values of partial regression coefficient (B), the higher the CT grade was, the greater the risk of SIRS was. The risk of SIRS was 4.472 times higher as each increasement of the grade (Table 5).

\section{Discussion}


Obstruction of upper urinary tract by calculi can cause urine accumulation, lead to bacterial proliferation and kidney infections. This may further lead to rapid destruction of kidney tissue structure. In recent years, the diagnostic accuracy of CT examination for calculi has reached 95\% 100\% [12], and it has been used as a routine preoperative examination of calculi patients. However, the diagnosis for kidney infection by CT has not been paid much attention in clinical practice. Therefore, this study aims to at establish a CT imaging grading system and analyze its clinical value in evaluating upper urinary tract calculi with kidney infection.

The normal intrapelvic pressure is $4-10 \mathrm{~cm} \mathrm{H} 2 \mathrm{O}$. In the early stage of acute ureteral obstruction, the renal pelvis and calves are dilated and stagnant, manifested by the thickening of the renal pelvis and calves and the slight expansion of the renal pelvis and calves on CT images [13]. When the pressure continues to rise, the effusion will enter the renal sinus through the calyx. In plain CT scan, the fat in renal sinus is difficult to be distinguished from renal parenchyma [14], especially during edema, both manifesting as thickening of renal parenchyma. Only when fluid accumulated within the renal sinus at the renal hilum can the separation of renal sinus edema and vague fat space be found in CT scan. In this study, patients with dilation and hydrops of the renal pelvis and calyces, thickened walls of renal pelvis and calyces, and visible obese fat space were classified as CT grade 1 .

When pressure in the renal pelvis is significantly increased, urine containing pathogenic bacteria and inflammatory cells may infiltrate from the renal interstitium or lymphatic vessels into the subrenal capsule or perirenal space, causing thickened and coarse kidney capsules, increased density of the perirenal space and thickened renal fascias [15]. According to studies from Raptopoulos and other scholars [16], there are multi-layers of fat space in the perirenal space, which are called bridge septa. The function of bridge septa is mainly to buffer the kidney pressure, and to drain fluid, pus and urine beneath the renal capsule and the renal sinus. Under normal circumstances, it is difficult for CT scan to identify the bridge septa, but there is thickening on CT if the bridge septa drainage results in increased hydrops during perirenal space edema [17]. Therefore, we defined patients with CT manifestations of inflammatory exudation of the renal capsule, increased density of the interrenal fat space, and thickened renal fascia as CT grade 2. The reasons are considered as follows: pathogenic bacteria and inflammatory cells may spread to the perirenal fascia through renal interstitial and lymphatic vessels. Renal parenchyma may be not yet involved, with less blood supply to the perirenal fascia. Thus, pathogenic bacteria and inflammatory cells are less likely to enter other body parts. The results of this study showed that the clinical symptoms and rise of inflammatory indicators of patients in CT grade 2 did not increase significantly.

Hydrops in the renal pelvis and calyces increases, and the pressure increases accordingly, therefore, urine within the renal sinus further enters the renal tubules through the corresponding renal papilla of the renal calyces, thus reversely flowing to the renal parenchyma [18]. Acute inflammatory reactions occur in the renal parenchyma, showing aggregation and infiltration of inflammatory cells, telangiectasia, edema of localized or patchy tissues, presence of edema and slight ischemic areas and presence of diffuse or punctate distribution [19]. On plain CT images, these features are manifested as kidney enlargement, 
thickened renal parenchyma, decreased density, and unclear decomposition of renal cortex and medulla $[19,20]$. Multiple abscesses or infarct focuses are visible, characterized by one or more wedge-shaped banded low-density focuses extending from the renal papilla to the renal cortex, in striated signs $[19,20]$. Thus, we classified the patients with CT manifestations of local thickening of the renal parenchyma, decreased density, unclear boundary of the renal cortex medulla, and local wedge swelling of the renal parenchyma and low-density foci as CT grade 3. Emphysematous pyelonephritis or renal abscess may occur with the aggravation of pathological changes or because of the highly toxic pathogenic bacteria [21]. Emphysematous pyelonephritis is a rare urological emergency, presenting with inflammatory necrosis of acute renal parenchyma and perirenal tissues, and characteristic emphysematous of renal parenchyma, perirenal tissues, and collecting systems [22]. Emphysematous pyelonephritis is a fatal disease, and if not diagnosed and treated in a timely manner, it may rapidly develop to septic shock, with a mortality rate of over $50 \%$ [23]. Therefore, in this study, renal abscess and emphysema pyelonephritis were classified as CT grade 4 .

Paick SH et al [24] classified CT images into 4 grades according to renal parenchymal involvement, including no renal parenchymal involvement (grade1), less than $25 \%$ involvement (grade 2), $25 \%$ or greater involvement but less than $50 \%$ involvement (grade 3), and $50 \%$ or greater involvement (grade 4 ). The higher the grade was, the more severe the clinical symptoms were. However, the grading system was established based on the CT enhancement scan. Numerous patients with upper urinary tract calculi are complicated with fever and are not suitable for injection of contrast agents. Thus, CT enhancement scan was not possible in these patients. In addition, pathogenic bacteria and inflammatory cells could infiltrate into subrenal capsules or perirenal space through renal interstitium or lymphatic vessels, which were not included in their grading system. In this study, plain CT scan was adopted, which was easy to be carried out in clinical practice. Plain CT images of upper urinary tract calculi with kidney infection were collected and analyzed, and corresponding grading system was established. According to CT images of renal pelvis, renal parenchyma and perirenal involvement, CT was classified into 4 grades. Finally, based on the CT grades, we compared the clinical manifestations, laboratory tests, and prognostic outcomes of patients at different levels.

Urinary leukocyte is an important indicator of the inflammatory response after bacterial infection in urine [25]. The results of our study showed that urine-leukocyte-positive patients had higher CT grade. The reason may be that the more inflammatory mediators and inflammatory cells caused by pathogenic bacteria in the urine, the more they flow back into the renal parenchyma, and the more severe the damage to the renal parenchymal structure. However, some of the patients with CT grade 4 did not have leukocytes in the urine. We suppose that this may because of the complete obstruction of urethra by stones. Nevertheless, such patients often were in more serious conditions. The positive urine culture results among different grades showed no statistical significance, which may be due to small sample size and the small number of urine-positive samples of various grades. Koh et al [26] found that diabetes was a high-risk factor for incidence of patients with urinary sepsis, and pathogenic bacteria were more likely to reproduce in the environment of high blood glucose. The results of the study showed that patients with diabetes were in higher CT grade. 
The CT value of the substance reflects the density of the substance, that is, the higher the CT value, the higher the density. When pathogenic bacteria, inflammatory mediators and inflammatory cells involve the kidney, the density of the involved parenchymal kidney decreases, and the CT value decreases correspondingly [27]. To avoid individual difference, the ratio of CT values of the affected side and that of the healthy side was calculated in this study to compare the CT value differences of patients with different CT grades. The results showed that the lower the ratio of CT values of the affected kidney cortex to that of the healthy kidney cortex was, the higher the CT grade was, and the difference was statistically significant. The Eta correlation analysis showed that CT1 was correlated with CT grading with Eta coefficients all higher than 0.7 , that is, the ratio of CT values of the affected renal cortex to that of the healthy renal cortex was negatively correlated with the CT grading. With high degree of correlation, the lower the ratio was, the higher the CT grades were. Urine containing pathogenic bacteria accumulates in the renal pelvis and it produces an inflammatory response, resulting in an increase of pathogenic bacteria, inflammatory mediators and inflammatory cells, as well as an increase in density, and a corresponding increase in CT values of the renal pelvis [28]. The results of this study showed that the ratio of $\mathrm{CT}$ values of the affected renal pelvises with those of the healthy renal pelvises were different in patients in different CT grades.

When there are pathogenic bacteria, the kidneys produce an inflammatory response under the stimulation of pathogenic bacteria and their toxic products. In addition, pathogens and their toxic products may directly enter the circulatory system through the renal parenchyma, thereby causing a systemic inflammatory response and increased production of inflammation indicators [29, 30]. It is found that CT images accurately show the degree of kidney infection, and that patients with more severe kidney infections have more severe clinical symptoms [31]. Our results showed that the blood white blood cell count, blood neutrophil ratio, CRP and PCT of patients with different CT grades were significantly different. The correlation analysis results of Eta coefficient also showed that the Eta coefficients of CRP and CT grades were higher than 0.7 , indicating a high degree of correlation. The higher the CT grade, the more severe the kidney infection, and the higher the CRP index of systemic inflammation. The univariate analysis results showed that CRP was a risk factor for SIRS. CRP may be used as a reference index for predicting SIRS at early stage.

With the presence or absence of SIRS as the end point of observation, univariate and multivariate analysis was conducted on the risk factors influencing the occurrence of SIRS in patients with upper urinary tract calculi. Univariate analysis results showed significant differences in CT1, CT2, CRP, PCT, neutrophil ratio and CT grading between the SIRS group and the non-SIRS group. Multivariate analysis results showed that only $\mathrm{CT}$ grading was statistically significant, which was an independent factor influencing SIRS. According to the values of partial regression coefficient (B), the higher the CT grades were, the greater the risk of SIRS was. The risk of SIRS was 4.472 times higher as each increasement of the grade. Therefore, results of the study showed that the higher the CT grade was, the greater the risk of SIRS in patients with upper urinary tract calculi were, which provides clinical guidance for early clinical diagnosis and treatment of SIRS. Kim et al. showed that CT image grading could be used to predict the severity and course of disease [32]. The higher the CT grades were, the worse the clinical symptoms and 
the prognosis were [32]. By CT grading, we evaluated the degree of kidney infection and our results may better guide the treatment. For severe kidney infection caused by upper urinary tract calculi, priority should be given to the removal of obstruction and effusion drainage before the second stage of stone treatment until infection control.

This study has some limitations. For example, the retrospective study and small sample size may affect the true level of CT grading and disease severity changes. Thus, results of the study need further verification by multi-center and large-sample clinical data.

In conclusion, CT images may reflect the involvement and scope of upper urinary tract calculi with kidney infection. The classification system based on CT imaging features has important clinical practical value. The higher the CT grade is, the more severe the clinical symptoms are and the greater the risk of SIRS is.

\section{Abbreviations}

\begin{tabular}{ll} 
SIRS & Systemic inflammatory response syndrome \\
\hline CRP & C-reactive protein \\
\hline PCT & procalcitonin \\
\hline SD & standard deviation
\end{tabular}

\section{Declarations}

\section{Ethics approval and consent to participate}

The clinical study conformed to the relevant requirements of World Medical Association Declaration of Helsinki. The study was approved by the ethics review board of the 180th Hospital of PLA.

\section{Consent for publication}

Written informed consents were obtained from every patient.

\section{Availability of data and materials}

The datasets used and/or analysed during the current study are available from the corresponding author on reasonable request.

\section{Competing interests}

The authors declare that they have no competing interests.

\section{Funding}

This work was supported by 2017 Quanzhou Science and Technology Project Fund (No. 2018Z143). 


\section{Authors' contributions}

Study Design: Jianping Zhang, Rongkai Lin

Data Collection: Jianping Zhang, Chaoxiong Deng, Xiaoxia Wu

Statistical Analysis: Rongkai Lin, Xiaoxia Wu

Data Interpretation: Jianping Zhang, Xiaoxia Wu

Manuscript Preparation: Jianping Zhang

Literature Search: Chaoxiong Deng, Chaoyu Yang

Funds Collection: Jianping Zhang, Rongkai Lin

\section{Acknowledgements}

Not applicable

\section{References}

1. Bonkat G, Cai T, Veeratterapillay R, Bruyère F, Bartoletti R, Pilatz A, et al. Management of Urosepsis in 2018. Eur Urol Focus. 2019;5(1):5-9.

2. Marchini GS, Vicentini FC, Mazzucchi E, Brito A, Ebaid G, Srougi M. Silent ureteral stones: impact on kidney function--can treatment of silent ureteral stones preserve kidney function? Urology. 2012;79(2):304-8.

3. Roberson NP, Dillman JR, Reddy PO, DeFoor W Jr, Trout AT. Ultrasound versus computed tomography for the detection of ureteral calculi in the pediatric population: a clinical effectiveness study. Abdom Radiol (NY). 2019;44(5):1858-66.

4. Danilovic A, Rocha BA, Torricelli FCM, Marchini GS, Batagello C, Vicentini FC, et al. Size is Not Everything That Matters: Preoperative CT Predictors of Stone Free After RIRS. Urology. 2019;132:638.

5. Boissier R, Baboudjian M, Di Bisceglie M. Evaluation of the impact of seric procalcitonin in the management of obstructive acute pyelonephritis. European Urology Supplements. 2019;18(1):e534.

6. Stalenhoef JE, van Nieuwkoop C, Wilson DC, van der Starre WE, van der Reijden TJK, Delfos NM, et al. Procalcitonin, mid-regional proadrenomedullin and C-reactive protein in predicting treatment outcome in community-acquired febrile urinary tract infection. BMC Infect Dis. 2019;19(1):161.

7. Sharma G, Tarafdar S, Merchant S. Urography: Various Imaging Modalities. Is CT Urography most promising successor of traditional IVU. International Journal of Scientific Research. 2016;5:65.

8. Farrell MR, Papagiannopoulos D, Ebersole J, White G, Deane LA. Perinephric Fat Stranding Is Associated with Elevated Creatinine Among Patients with Acutely Obstructing Ureterolithiasis. J 
Endourol. 2018;32(9):891-5.

9. Coll DM, Varanelli MJ, Smith RC. Relationship of spontaneous passage of ureteral calculi to stone size and location as revealed by unenhanced helical CT. AJR Am J Roentgenol. 2002;178(1):101-3.

10. Parakh A, Lee H, Lee J, Eisner B, Sahani D, Do S. Urinary Stone Detection on CT Images Using Deep Convolutional Neural Networks: Evaluation of Model Performance and Generalization. Radiology: Artificial Intelligence. 2019;1:e180066.

11. Liu D, Wu J, Chen S, Liu Y, Zhang G, Ping H, et al. Ultrasonography Can Replace CT Scans as the Initial Imaging Examination of Ureteral Calculi. Urol Int. 2019;103(1):68-73.

12. Moon JW, Park BK, Kim CK, Park SY. Evaluation of virtual unenhanced CT obtained from dual-energy CT urography for detecting urinary stones. Br J Radiol. 2012;85(1014):e176-81.

13. Jung H, Osther PJ. Intraluminal pressure profiles during flexible ureterorenoscopy. Springerplus. 2015;4:373.

14. Sokhi HK, Mok WY, Patel U. Stage T3a renal cell carcinoma: staging accuracy of CT for sinus fat, perinephric fat or renal vein invasion. Br J Radiol. 2015;88(1045):20140504.

15. Ogreden E, Oguz U, Demirelli E, Benli E, Özen Ö. The impact of ureteral Double-J stent insertion following ureterorenoscopy in patients with ureteral stones accompanied by perirenal fat stranding. Arch Ital Urol Androl. 2018;90(1):15-9.

16. Raptopoulos V, Touliopoulos P, Lei QF, Vrachliotis TG, Marks SC, Jr. Medial border of the perirenal space: CT and anatomic correlation. Radiology. 1997;205(3):777-84.

17. Aizenstein RI, Wilbur AC, O'Neil HK. Interfascial and perinephric pathways in the spread of retroperitoneal disease: refined concepts based on CT observations. AJR Am J Roentgenol. 1997;168(3):639-43.

18. Qian C, Tan B, Chen Y. The relationship between determination of RIRS renal pelvis pressure and postoperative fever. Int J Clin Exp Med. 2018;11(8):8634-9.

19. Cheng $\mathrm{CH}$, Tsau YK, Lin TY. Is acute lobar nephronia the midpoint in the spectrum of upper urinary tract infections between acute pyelonephritis and renal abscess? J Pediatr. 2010;156(1):82-6.

20. Taniguchi LS, Torres US, Souza SM, Torres LR, D'Ippolito G. Are the unenhanced and excretory CT phases necessary for the evaluation of acute pyelonephritis? Acta Radiol. 2017;58(5):634-40.

21. Hamouda M, Aloui S, Skhiri H, Letaif A, Frih MA, Ben Dhia N, et al. Emphysematous pyelonephritis in a diabetic patient on maintenance hemodialysis: a case report. Arab J Nephrol Transplant. 2014;7(2):109-11.

22. Deoraj S, Zakharious F, Nasim A, Missouris C. Emphysematous pyelonephritis: outcomes of conservative management and literature review. BMJ Case Rep. 2018;2018.

23. Aboumarzouk OM, Hughes O, Narahari K, Coulthard R, Kynaston H, Chlosta P, et al. Emphysematous pyelonephritis: Time for a management plan with an evidence-based approach. Arab J Urol. 2014;12(2):106-15. 
24. Paick SH, Choo GY, Baek M, Bae SR, Kim HG, Lho YS, et al. Clinical value of acute pyelonephritis grade based on computed tomography in predicting severity and course of acute pyelonephritis. $\mathrm{J}$ Comput Assist Tomogr. 2013;37(3):440-2.

25. Cai T, Lanzafame P, Caciagli P, Migno S, Mereu L, Mattevi D, et al. Role of increasing leukocyturia for detecting the transition from asymptomatic bacteriuria to symptomatic infection in women with recurrent urinary tract infections: A new tool for improving antibiotic stewardship. Int J Urol. 2018;25(9):800-6.

26. Koh GC, Peacock SJ, van der Poll T, Wiersinga WJ. The impact of diabetes on the pathogenesis of sepsis. Eur J Clin Microbiol Infect Dis. 2012;31(4):379-88.

27. Georgiades CS, Moore CJ, Smith DP. Differences of renal parenchymal attenuation for acutely obstructed and unobstructed kidneys on unenhanced helical CT: a useful secondary sign? AJR Am J Roentgenol. 2001;176(4):965-8.

28. Yuruk E, Tuken M, Sulejman S, Colakerol A, Serefoglu EC, Sarica K, et al. Computerized tomography attenuation values can be used to differentiate hydronephrosis from pyonephrosis. World J Urol. 2017;35(3):437-42.

29. Claessens YE, Trabattoni E, Grabar S, Quinquis L, Der Sahakian G, Anselmo M, et al. Plasmatic presepsin (sCD14-ST) concentrations in acute pyelonephritis in adult patients. Clin Chim Acta. 2017;464:182-8.

30. Qin Y, Liu J, Liu J, Hu F. Collectins in urinary tract and kidney diseases. Int Urol Nephrol. 2018;50(4):695-703.

31. Lim SK, Ng FC. Acute pyelonephritis and renal abscesses in adults--correlating clinical parameters with radiological (computer tomography) severity. Ann Acad Med Singapore. 2011;40(9):407-13.

32. Kim JS, Lee S, Lee KW, Kim JM, Kim YH, Kim ME. Relationship between uncommon computed tomography findings and clinical aspects in patients with acute pyelonephritis. Korean J Urol. 2014;55(7):482-6.

\section{Tables}


Table 1. Comparison of general data of patients with different CT grades.

\begin{tabular}{|c|c|c|c|c|c|c|}
\hline & \multicolumn{4}{|c|}{ CT Grades } & \multirow[t]{2}{*}{$\mathrm{F}$} & \multirow[t]{2}{*}{$\mathrm{P}$} \\
\hline Indicators & Grade $1(n=48)$ & Grade $2(n=30)$ & Grade $3(n=24)$ & Grade $4(n=24)$ & & \\
\hline Age (Years Old) & $54.54 \pm 6.19$ & $56.07 \pm 7.85$ & $51.17 \pm 13.36$ & $54.58 \pm 11.96$ & 0.622 & 0.60 \\
\hline Sex (Male/Female) & $26 / 22$ & $10 / 20$ & $6 / 18$ & $8 / 16$ & 7.050 & 0.06 \\
\hline Side (Left/Right) & $10 / 38$ & $10 / 20$ & $6 / 18$ & $10 / 14$ & 3.908 & 0.27 \\
\hline Stone position (Upper/Middle/Lower ureter) & $40 / 5 / 3$ & $18 / 2 / 10$ & $16 / 2 / 6$ & $14 / 4 / 6$ & 11.439 & 0.07 \\
\hline
\end{tabular}


Table 2. Comparison of clinical symptoms and physical examination results of patients with different CT grades.

\begin{tabular}{|c|c|c|c|c|c|c|}
\hline \multirow[t]{2}{*}{ Indicators } & \multicolumn{4}{|c|}{ CT Grades } & \multirow[t]{2}{*}{$\mathrm{F} / \mathrm{C}^{2}$} & \multirow[t]{2}{*}{$\mathrm{P}$} \\
\hline & Grade 1 (48) & Grade 2 (30) & Grade 3 (24) & Grade $4(24)$ & & \\
\hline Renal Colic (with/without) & $42 / 6$ & $26 / 4$ & $22 / 2$ & $20 / 4$ & 0.856 & 0.860 \\
\hline Fever (with/without) & $0 / 48$ & $2 / 28$ & $12 / 12$ & $20 / 4$ & 69.155 & $<0.001^{*}$ \\
\hline Diabetes (with/without) & $28 / 20$ & $4 / 26$ & $2 / 22$ & $6 / 18$ & 26.929 & $<0.001^{*}$ \\
\hline Midstream Urine Culture (Positive/Negative) & $6 / 42$ & $2 / 28$ & $4 / 20$ & $6 / 14$ & 5.112 & 0.160 \\
\hline Blood Culture (Positive/Negative) & $2 / 46$ & $2 / 28$ & $2 / 22$ & $4 / 20$ & 3.337 & 0.311 \\
\hline Urine Leukocytes (Positive/Negative) & $40 / 8$ & $12 / 18$ & $10 / 14$ & $18 / 6$ & 21.598 & $<0.001^{*}$ \\
\hline Blood WBC Counts $\left(* 10^{9} / \mathrm{L}\right)$ & $8.00+1.21$ & $10.82+4.10$ & $15.66+8.97$ & $16.01+11.03$ & 5.92 & $0.001^{*}$ \\
\hline CT1 & $1.01 \pm 0.06$ & $0.85 \pm 0.14$ & $0.51 \pm 0.15$ & $0.49 \pm 0.23$ & 53.462 & $<0.001^{*}$ \\
\hline CT2 & $0.5(0.5,2)$ & $5.5(3,9)$ & $4(2.2,5.8)$ & $4.9(2.1,10.1)$ & 25.025 & $<0.001 *$ \\
\hline Maximum Body Temperature $\left({ }^{\circ} \mathrm{C}\right)$ & $36.7(36.4,37)$ & $36.7(36.4,36.8)$ & $37.7(36.9,39.4)$ & $39.3(37.8,40.2)$ & 21.266 & $<0.001 *$ \\
\hline Disease Time (Days) & $1(1,13)$ & $1(0.4,1)$ & $2.5(1,7.25)$ & $3(1,7)$ & 8.712 & $0.033^{*}$ \\
\hline Blood Neutrophil Percent(\%) & $63.5(57.3,68.7)$ & $80.3(51.8,85.1)$ & $81.2(65.6,91.5)$ & $84.1(70.4,92.5)$ & 14.146 & $0.003^{*}$ \\
\hline $\mathrm{CRP}(\mathrm{mg} / \mathrm{L})$ & $4(2.3,7.7)$ & $5.3(3,21.4)$ & $97.9(22.4,171)$ & $138(86.7,216)$ & 34.849 & $<0.001^{*}$ \\
\hline PCT(ug/L) & $0.01(0.01,0.01)$ & $0.01(0.01,0.05)$ & $1.7(0.1,20)$ & $3.4(0.25,79.1)$ & 37.948 & $<0.001 *$ \\
\hline Stone Size $(\mathrm{cm})$ & $1.9(1.9,2.15)$ & $1(1,1.5)$ & $1.75(1.5 \square 2.5)$ & $2.9(1.5,3.6)$ & 15.435 & $0.001 *$ \\
\hline
\end{tabular}

Notes: ${ }^{*} \mathrm{P}<0.05$. CRP, C-reactive protein; PCT, procalcitonin; CT1, the ratios of renal cortex CT values on the affected side and those on the healthy side; $\mathrm{CT} 2$, the ratios of renal pelvis $\mathrm{CT}$ values on the affected side and those on the healthy side.

Table 3. Correlation analysis results of some indicators and CT grade.

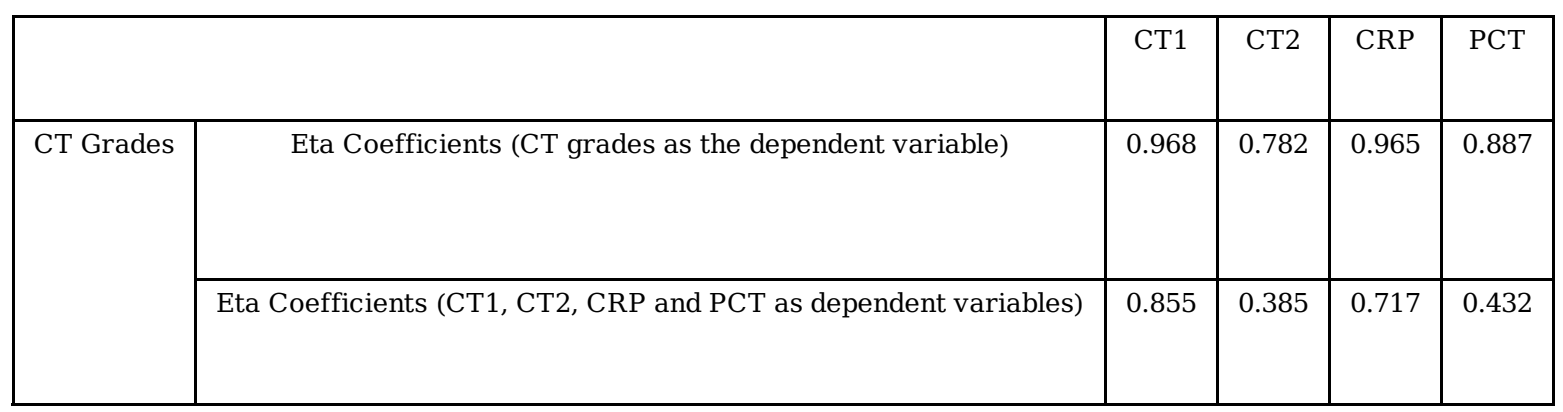

Notes: CRP, C-reactive protein; PCT, procalcitonin; CT1, the ratios of renal cortex CT values on the affected side and those on the healthy side; $\mathrm{CT} 2$, the ratios of renal pelvis $\mathrm{CT}$ values on the affected side and those on the healthy side.

Table 4. Univariate analysis of SIRS in patients with upper urinary calculi. 


\begin{tabular}{|c|c|c|c|c|}
\hline \multirow[t]{2}{*}{ Indicators } & \multicolumn{2}{|c|}{ SIRS } & \multirow[t]{2}{*}{$\mathrm{Z} / \mathrm{t} / \mathrm{c} 2$} & \multirow[t]{2}{*}{$\mathrm{P}$} \\
\hline & Yes & No & & \\
\hline CT1 & $0.50(0.38,0.63)$ & $0.96(0.83,1.00)$ & -4.362 & $<0.001^{*}$ \\
\hline CT2 & $5.50(2.57,8.25)$ & $2.00(0.50,5.25)$ & -2.781 & $0.005^{*}$ \\
\hline Stone Size & $1.80(1.50,2.90)$ & $1.90(1.50,2.23)$ & -0.618 & 0.537 \\
\hline Disease Time & $3.00(1.00,7.50)$ & $1.00(1.00,7.00)$ & -1.574 & 0.116 \\
\hline CRP & $132.00(88.45,188.50)$ & $5.80(2.90,21.60)$ & -4.809 & $<0.001^{*}$ \\
\hline PCT & $5.61(0.29,35.94)$ & $0.01(0.01,0.05)$ & -4.959 & $<0.001^{*}$ \\
\hline Blood Culture & $2.00(2.00,2.00)$ & $2.00(2.00,2.00)$ & -1.574 & 0.115 \\
\hline Urine Culture & $2.00(1.00,2.00)$ & $2.00(2.00,2.00)$ & -1.773 & 0.076 \\
\hline Proportion of Blood Neutrophil Percent & $81.51 \pm 13.27$ & $69.15 \pm 13.69$ & 3.205 & $0.002 *$ \\
\hline Grades (Grades 1/2/3/4) & $0 / 2 / 12 / 20$ & $48 / 28 / 12 / 4$ & 69.155 & $<0.001 *$ \\
\hline Urine Leukocyte (Positive/Negative) & $20 / 14$ & $60 / 32$ & 0.438 & 0.508 \\
\hline Diabetes (with/without) & $8 / 26$ & $32 / 60$ & 1.451 & 0.228 \\
\hline Renal Colic (with/without) & $18 / 6$ & $82 / 10$ & 2.118 & 0.146 \\
\hline
\end{tabular}

Notes: ${ }^{*} \mathrm{P}<0.05$. CRP, C-reactive protein; PCT, procalcitonin; CT1, the ratios of renal cortex CT values on the affected side and those on the healthy side; CT2, the ratios of renal pelvis CT values on the affected side and those on the healthy side; SIRS, systemic inflammatory response syndrome.

Table 5. Multivariate analysis of SIRS in patients with upper urinary calculi. 


\begin{tabular}{|c|c|c|c|c|c|c|c|c|}
\hline Independent Variables & B & S.E. & Wald & df & P & Exp(B) & \multicolumn{2}{|c|}{$95 \%$ C.I. for EXP(B) } \\
\cline { 6 - 9 } & & & & & & & Lower & Upper \\
\hline CT1 & & & & & & & & \\
\hline CT2 & 0.025 & 0.070 & 0.130 & 1 & 0.719 & 1.026 & 0.894 & 1.177 \\
\hline Proportion of Neutrophil & -0.007 & 0.046 & 0.020 & 1 & 0.887 & 0.993 & 0.907 & 1.088 \\
\hline CRP & 0.013 & 0.009 & 1.996 & 1 & 0.158 & 1.013 & 0.995 & 1.032 \\
\hline PCT & 0.004 & 0.017 & 0.064 & 1 & 0.800 & 1.004 & 0.971 & 1.039 \\
\hline Urine Culture & 0.562 & 1.385 & 0.165 & 1 & 0.685 & 1.754 & 0.116 & 26.468 \\
\hline CT Grading & 1.498 & 0.743 & 4.060 & 1 & $0.044^{*}$ & 4.472 & 1.042 & 19.201 \\
\hline Constant & -5.751 & 5.498 & 1.094 & 1 & 0.296 & 0.003 & & \\
\hline
\end{tabular}

Notes: * $\mathrm{P}<0.05$. CRP, C-reactive protein; PCT, procalcitonin; CT1, the ratios of renal cortex CT values on the affected side and those on the healthy side; CT2, the ratios of renal pelvis CT values on the affected side and those on the healthy side.

\section{Figures}

A

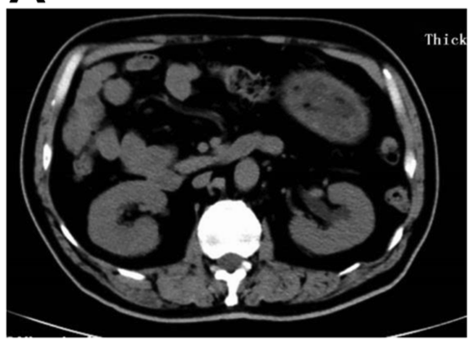

B

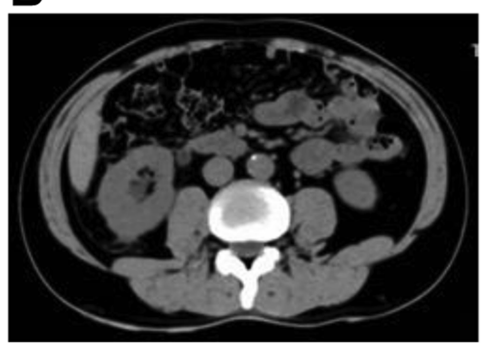

C

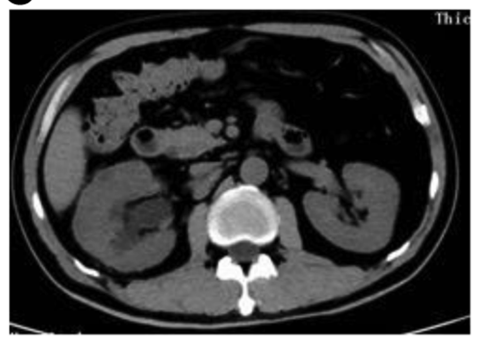

D

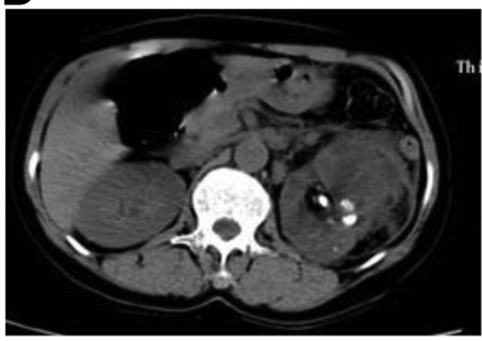

Figure 1

CT grading of upper urinary tract calculi with kidney infection. (A) CT grade 1 ; (B) CT grade 2; (C) CT grade 3; and (D) CT grade 4. 